\title{
QUANTIFICATION OF PHASES IN CEMENTS AND CLINKER SAMPLES BY THE RIETVELD METHOD USING THE TOPAS AND TOPAS BBQ SOFTWARES
}

\author{
da Silva, R. F.*
}

Application Specialist. AXS Division, Bruker do Brasil, Atibaia, São Paulo, Brasil

*renato.silva@bruker.com

X-ray diffraction (XRD) allows the process control and quality of the raw material, hot meal, clinker and cement, based on the real phase composition, whose informations can not be obtained by other analytical techniques. The quantitative analysis by Rietveld Method of these materials using TOPAS software allows to see trends and correlations in the cement process, because makes possible a direct and precise phase analysis. The TOPAS software is a dedicated program for researchers while the TOPAS BBQ is dedicated to industry and automatically performs quantitative analysis of phases from the XRD data without any user intervention. The purpose of this work is the phase quantification of clinker and cement samples by the Rietveld Methold using the TOPAS and the TOPAS BBQ softwares. The samples were grinded and analyzed in a Bruker D8 Advance Diffractomer and a Bruker D2 PHASER, respectively. The TOPAS software was used to quantify the different samples. The results for all the samples showed an excellent adjustment between the experimental and the calculated curve. The Rietveld quantitative phase analysis by TOPAS and TOPAS BBQ software are a powerful tools to investigate the composition of cement and clinkers with a high accuracy and enables the process control and quality of these materials, based on their real composition. 